\title{
Papers
}

\section{Outbreak of severe acute respiratory syndrome in a tertiary hospital in Singapore, linked to an index patient with atypical presentation: epidemiological study}

Khuan Yew Chow, Chien Earn Lee, Moi Lin Ling, Derrick Mok Kwee Heng, Soon Ghee Yap

\begin{abstract}
Objective To describe an outbreak of severe acute respiratory syndrome (SARS) in a tertiary hospital in Singapore, linked to an index patient with atypical presentation, and the lessons learnt from it.

Design Descriptive study.

Setting A tertiary hospital in Singapore.

Participants Patients, healthcare workers, and visitors who contracted SARS in Singapore General Hospital.

Main outcome measures Probable SARS as defined by the World Health Organization.

Results The index patient presented with gastrointestinal bleeding, initially without changes to his chest radiograph. Altogether 24 healthcare workers, 15 patients, and 12 family members and visitors were infected. The incubation period ranged from three to eight days. Only 13 patients were isolated on their dates of onset.

Conclusions Atypical presentation of SARS infection must be taken into consideration when managing patients with a history of contact with SARS patients. The main gap in the containment strategy in this outbreak was the failure to identify the index patient as someone who had been discharged from a ward in another hospital that managed probable SARS cases. Strict infection control measures, a good surveillance system, early introduction of isolation procedures, and vigilant healthcare professionals are essential for controlling outbreaks.
\end{abstract}

\section{Introduction}

On 6 March 2003 the Ministry of Health in Singapore issued a press release that three Singaporeans had developed atypical pneumonia after travelling to Hong Kong. ${ }^{1}$ Around the same time, the World Health Organization issued a global health alert on severe acute respiratory syndrome (SARS), an atypical pneumonia that has been associated aetiologically with a novel coronavirus, SARS-COV. ${ }^{2}$ We describe an outbreak in a tertiary hospital delivering acute care in Singapore that was linked to an index patient with atypical presentation and highlight the lessons learnt in managing the outbreak.

\section{Methods}

We used data extracted from the period of 24 March to 15 April 2003 to prepare this report. We describe the epidemiological link of 51 patients infected directly or indirectly by an index patient in Singapore General Hospital, a tertiary hospital delivering acute care.
The epidemiology and contact tracing team interviewed patients and their relatives; extracted information on patients' movement from hospital databases; and reviewed the duty rosters of healthcare staff, patients' case records, temperature charts, and discharge summaries to obtain information that supported the chain of transmission.

An epidemiologist then analysed the information to identify the possible sources of infection and mapped the possible contacts on the basis of the following variables:

- Time: whether the patients, staff, or visitors contacted the infection within the incubation period

- Place: whether the patients, staff, or visitors were in the same place, where transmission of infection could have occurred

- Person: whether they were in contact with one another.

At the time of the outbreak no reliable laboratory test for SARS was available; hence we used a case definition for a probable case that was based on the initial definition from the World Health Organization. $^{3}$

\section{Results}

On 4 April 200313 staff from two surgical wards in Singapore General Hospital were discovered to have developed fever over the past four days. In the next two days all patients and healthcare staff from the two wards were transferred to Tan Tock Seng Hospital, the national centre for managing SARS patients, for further assessment and to ringfence the source of infection.

A registrar was the first healthcare worker to develop symptoms, and initial investigations were focused on her as the potential source. However, we were not able to trace the source of infection based on the available information.

Attention was then focused on identifying the index patient, in particular among the patients who had been transferred from Tan Tock Seng Hospital, who could have infected the registrar and other healthcare staff. This was a complex undertaking because the multiple medical problems of these patients might have masked the symptoms of SARS.

A breakthrough came when SARS was diagnosed in the brother of a patient on 8 April in the affected wards who visited the patient on the ward. The investigating team subsequently focused on this patient. Detailed epidemiological investigations resulted in the conclusion that this patient, who was admitted on 24 March to Singapore General Hospital, was the index patient source of the SARS outbreak at that hospital.

Description of the index case

The index patient, an elderly Chinese man with chronic renal disease and diabetes mellitus, had previously been admitted to 
the ward at Tan Tock Seng Hospital, where the first SARS patient in Singapore was treated. Subsequently he was admitted to a surgical ward in Singapore General Hospital on 24 March for gastrointestinal bleeding and a diabetes related foot ulcer on his right heel.

To determine the source of gastrointestinal bleeding he underwent gastroscopy, colonoscopy, and barium studies on 26 and 28 March and 1 April, respectively. He was monitored for bloody stools daily and given laxatives in preparation for the procedures. From 26 March to 2 April he underwent desloughing and subsequently debridement of his foot ulcer.

On admission he had an oral temperature of $37.4^{\circ} \mathrm{C}$, but his temperature spiked on 26 March. He had no respiratory symptoms. On 28 March his blood culture yielded Escherichia coli, and he was subsequently treated for $E$ coli bacteraemia. Ultrasound investigation of his kidneys showed a mass in his right kidney, reported as likely to be due to an abscess or tumour.

Multiple chest $\mathrm{x}$ ray films from admission to 2 April did not show any focal lesion in his lungs. A chest $\mathrm{x}$ ray film from 3 April showed ill defined shadowing of the air space in the right lower zone and left perihilar region. However, a chest $x$ ray film from 4 April showed no abnormalities.

The infectious disease doctor reviewed the patient on 2 April and thought that the kidney abscess had resulted in the secondary infection, $E$ coli bacteraemia. Treatment with intravenous imipenem-cilastatin was started immediately. As SARS infection could not be excluded the patient was transferred directly to an isolation ward on the same day. His fever settled on 4 April.

On 4 April specimens obtained by throat swab were sent for virological studies, but they were negative for respiratory viruses. It was only on 9 April, when epidemiological evidence was finally supported by definitive changes to his chest $\mathrm{x}$ ray film, that he was classed as a probable case of SARS.

\section{Description of the outbreak}

Twenty four healthcare workers, 15 patients, and 12 visitors, who were in either direct or indirect contact with the index patient, developed probable SARS. The index patient created five main clusters of infections.

The first cluster was that of healthcare workers in the two wards that he stayed before he was isolated. This group included a registrar, 13 nurses (all of whom had cared for the index patient), one healthcare attendant, and a radiographer who performed portable $\mathrm{x}$ rays on the ward.

The second cluster was a group of 12 patients from the two wards. Eight of these patients were directly infected by the index patient and the other four contracted secondary infections from two of the eight patients. Suspicion of an outbreak was not prompted at the early stage as most of the patients in the surrounding beds had multiple medical problems and were prone to infections. One of these patients was also suspected to have subsequently passed the infection to a surgeon who attended to him, although the surgeon was using full personal protective equipment. Seven visitors (excluding the family of the index patient who visited him) to the two wards also became infected.

The index patient also started a cluster of infection when he visited the diagnostic radiology department on 1 and 2 April. This included two healthcare assistants from the urology centre who were in the same waiting area as the index patient and two outpatients who attended the diagnostic radiology department for radiological procedures on 1 April. Similarly, a radiology healthcare assistant and a porter who had close contact with the index patient during the ultrasound procedure on 2 April were infected. Subsequently they infected a radiographer colleague. The index patient also infected the husband of another patient, who was waiting for his wife to undergo barium meal examination in the diagnostic radiology department, who subsequently infected his wife.

The fourth cluster of infection was at the national cancer centre. A radiographer from the diagnostic radiology department of the national cancer centre was infected when he came into indirect contact with the infected registrar. This radiographer subsequently infected a porter from the same department.

The fifth cluster of infection was the family of the index patient. Four of his family members developed the infection; the brother of the index patient had the earliest date of onset.

The SARS infections in Singapore General Hospital had also affected others in the community, but we included only only people infected in the hospital in our clusters. The index patient's brother and his sister in law infected two colleagues working in a wholesaler's, who subsequently infected their family members. As a result a total of 1825 people were placed on home quarantine. The porter working in the diagnostic radiology department at Singapore General Hospital infected eight of her friends through social contact.

Figure 1 shows the epidemiological links of the contacts of the index patients. The epidemic curve (fig 2) shows person to person spread during the outbreak. Close contacts between patients and staff and between staff had resulted in many staff in the two wards becoming infected around the same time and showing symptoms on the 4 April 2003.

We determined the incubation period of 15 patients and staff who had a single occasion of contact with the index patient. The incubation period ranged from three to eight days. Two thirds of this group had short incubation periods of three to four days (fig 3).

To determine the infectious period during which the infected patients and staff could have possibly transmitted their infection to others, we looked at the period between onset of symptoms to date of isolation for patients, healthcare staff, and infected visitors. Only 13 were isolated on or before their dates of onset of symptoms, the remaining 39 were isolated between one and 12 days from the onset of symptoms (fig 4 ).

\section{Discussion}

The atypical presentation of SARS has been documented previously. ${ }^{2}$ In addition to fever and respiratory symptoms, other common clinical features include anorexia (45-54.6\%) and diarrhoea $(25-27 \%) .{ }^{45}$ The patient in our study presented with fever, bloody stools, and leg ulcer but had no respiratory symptoms. Radiographic evidence of pneumonia was seen relatively late.

In the absence of respiratory symptoms the index patient was likely to have been shedding the SARS virus through his stool. His gastrointestinal bleed was probably a contributing factor as it required radiological and endoscopic examinations and daily monitoring of the stool colour. Associated procedures such as bowel preparation would also have increased viral shedding. Infection through contact with exudates from the index patient's foot ulcer could not be excluded either. Healthcare workers were at increased risk of exposure to the virus through transmission during contact. The fact that transmission had been limited to close contacts of the index patient implies that droplet spread or direct contact was the main mode of transmission in this outbreak.

The incubation period ranged from three to eight days; most of the patients and staff presented three to four days after expo- 


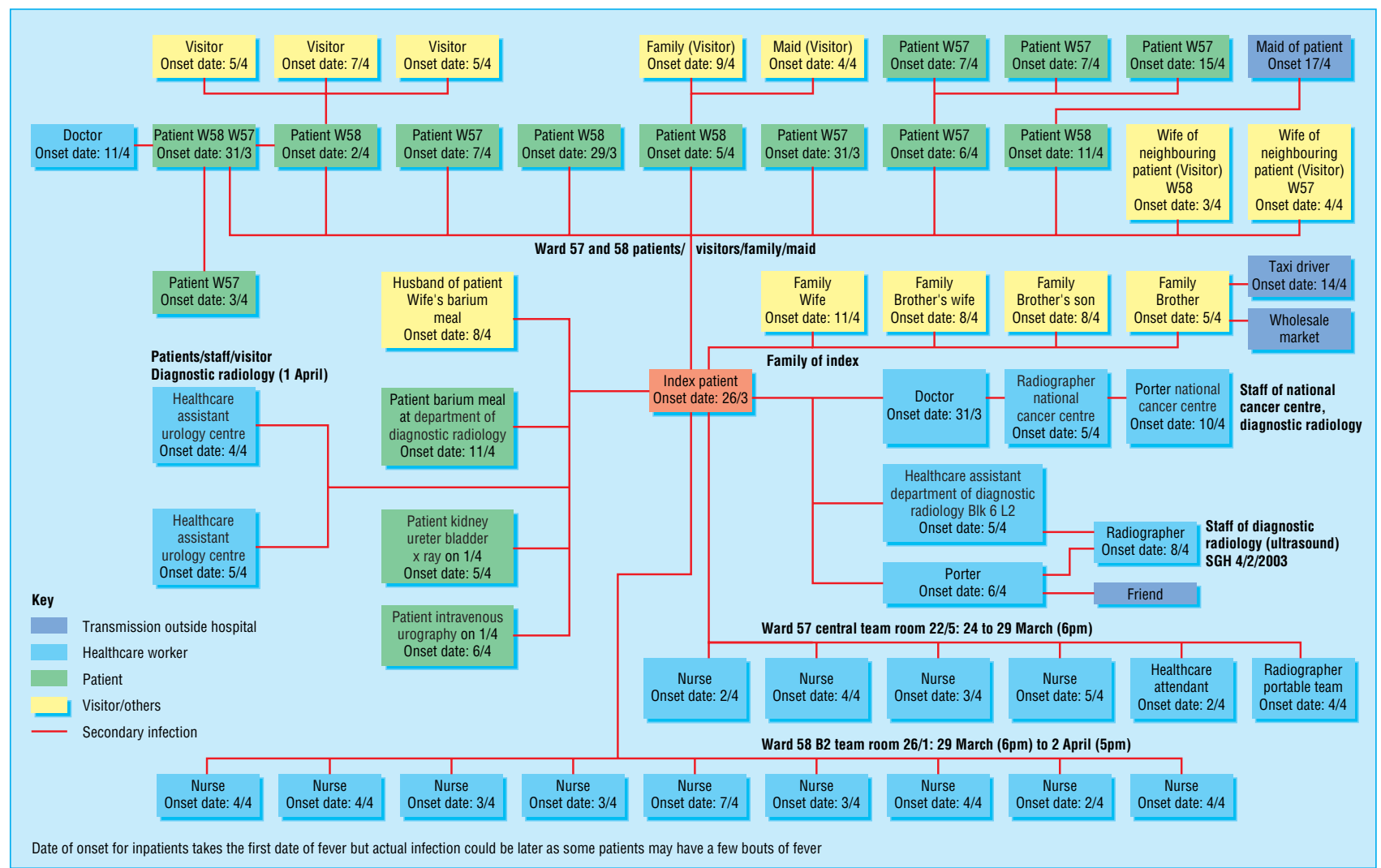

Fig 1 Map of contact tracing for the SARS cluster at Singapore General Hospital by SARS task force

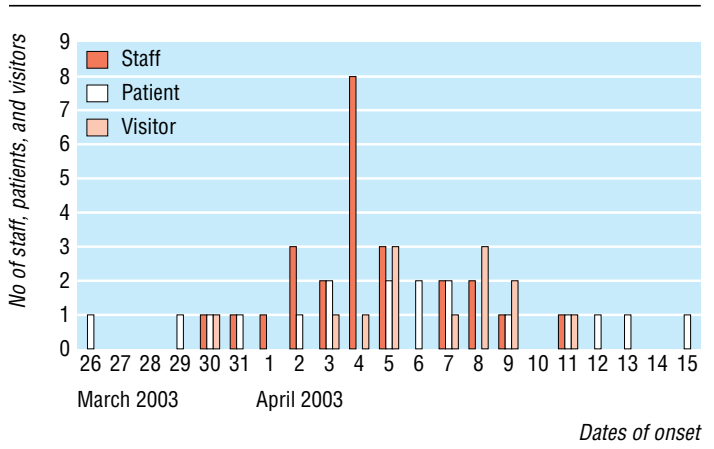

Fig 2 Epidemic curve of the SARS outbreak at Singapore General Hospital

sure. The computation of incubation period was straightforward for those with a single encounter with the index patient. It was more complex for people who had had multiple exposures, such as staff and patients.

The first people infected by the index patient began to present with fever from 29 March. Not until 4 April were numbers of symptomatic healthcare workers and patients sufficiently high to differentiate a definite cluster as opposed to "background noise." The index patient had already been isolated on 2 April as a precautionary measure, even before an epidemiological link was established. By then at least 42 of the 50 healthcare workers, patients, and visitors who were infected in Singapore General Hospital would have already have been infected.

With hindsight, draconian containment measures, such as quarantining all visitors to the affected wards as soon the fever cluster was detected on 4 April, might have prevented the infection from spreading beyond Singapore General Hospital, as the

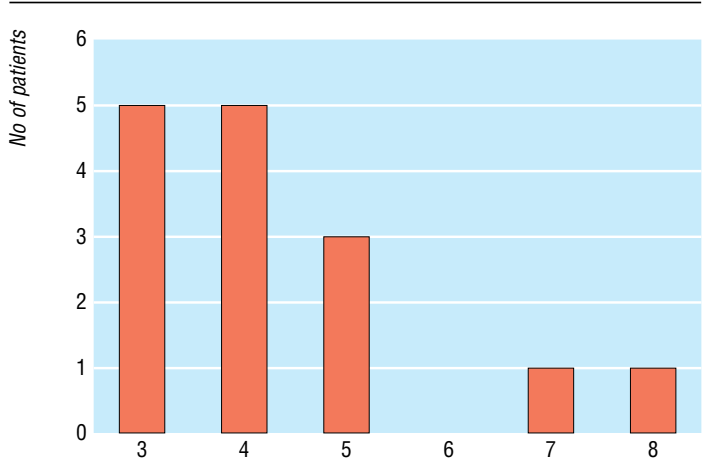

No of days

Fig 3 Incubation of probable SARS cases in the outbreak at Singapore General Hospital

visitor who started the cluster of infection at the wholesale centre showed symptoms only on the 5 April.

Personal protective equipment is another key element in the fight against SARS as the infection is highly transmissible and healthcare workers are particularly at risk. ${ }^{6-9}$ However, this may not offer full protection if healthcare workers do not follow the recommended infection control precautions. ${ }^{10}$ In addition to personal protective equipment Singapore General Hospital also introduced stringent infection control measures and organisational interventions (box), to ringfence the source of infection and prevent further transmission of infection.

Although these measures, if implemented from the onset, would have protected healthcare workers (assuming full compliance), some patients and visitors would still have been infected. 


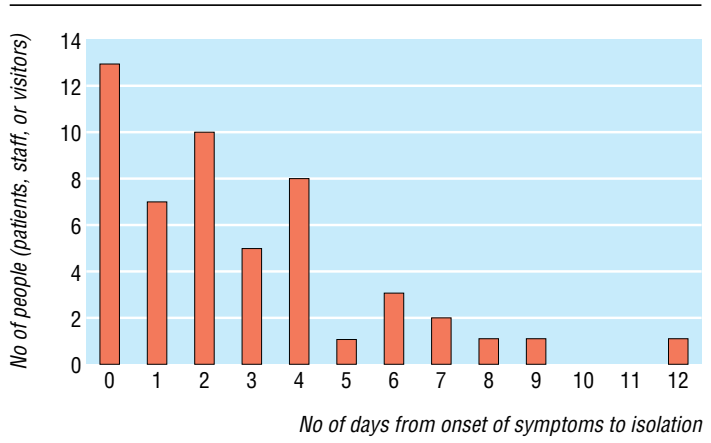

Fig 4 Infectious period of the probable SARS cases from Singapore General Hospital

These include patients who came into contact with the index patient at outpatient settings such as the radiology department.

\section{Conclusion}

The key lesson from this incident is the need to identify potentially infected patients before they enter the hospital's mainstream areas (wards etc) where patients, staff, and visitors mingle. Once our index patient had slipped through this gap, all

\section{Infection control measures}

- Early detection and prompt isolation of SARS cases in isolation rooms with negative pressure

- Hospital wide implementation of the use of personal protective equipment in all areas where care for patients is undertaken-use of N95 mask (mask that is $95 \%$ efficient at filtering out particles of sizes 0.3 micron and above), gowns, gloves, goggles, head covers, and handwashing

- Completion of SARS screening questionnaire and temperature screen before entry to areas where care for patients is undertaken

- All hospital staff to monitor their temperature three times a day

- Mandatory attendance for hospital staff on infection control measures against SARS and N95 mask fit test for all staff

- Intense contact tracing and mapping of cases and exposed people

- 10 day home quarantine for staff, patients, and visitors exposed to SARS patients

- 21 day home quarantine for staff discharged from SARS hospital (Tan Tock Seng Hospital)

\section{Organisational interventions}

Formation of SARS task force to formulate guidelines and implement intervention to control the outbreak

Restriction of visitors policy converted to a no visitor rule later Exposed patients and staff from affected wards transferred to Tan Tock Seng Hospital

Modular team systems for doctors and nurses

Suspension of elective services

Surveillance system to review patients transferred to Tan Tock Seng Hospital, staff with fever, and "hot" wards (wards from which patients were transferred to Tan Tock Seng Hospital for respiratory illnesses pending investigation for SARS or wards with clusters of febrile patients pending investigation for SARS Closure of ward (no admission, no discharge , no transfer) to prevent patients from being discharged till the source of infection is identified or patients in the ward cleared of SARS All sick staff must be treated and subsequently reviewed at staff clinic

\section{What is already known on this topic}

Severe acute respiratory syndrome (SARS) is an atypical pneumonia that has been associated aetiologically with a novel coronavirus

The disease originated in southern China and has spread globally

The clinical picture, treatment, and mode of transmission have been described

\section{What this study adds}

An outbreak in a tertiary hospital in Singapore was linked to a patient with atypical presentation

The hospital introduced special measures to control the outbreak

Strict infection control measures, a good surveillance system, early introduction of isolation procedures, and vigilant healthcare profession are essential for controlling such outbreaks

other containment measures were catching-up exercises. The identification of the index patient was complicated by the atypical presentation and the presence of comorbidities. Constant vigilance is needed, especially when managing immunocompromised patients (such as patients with chronic renal failure) with multiple medical problems.

Contributors: KYC wrote first draft of the paper. MLL, LCE, DMKH, and SGY subsequently edited the paper. MLL is the guarantor. All authors were actively involved in managing the outbreak.

Funding: None.

Competing interests: None declared.

Ethical approval: Professor Tay Boon Keng, chairman Medical Board and SARS Task Force Singapore General Hospital.

1 Ministry of Health. Severe acute respiratory syndrome. Press releases. http:// app.moh.gov.sg/sars/news/updates.asp (accessed 30 Dec 2003).

2 Centers for Disease Control and Prevention. Updated interim surveillance case definition for severe acute respiratory syndrome (SARS)-United States, April 29, 2003. MMWR Morb Mortal Wkly Rep 2003;52(17):391-3.

3 World Health Organization, Disease Outbreaks Reported. WHO issues emergency travel advisory: severe acute respiratory distress syndrome (SARS) spreads worldwide. http://www.who.int/csr/don/2003 03 15/en/ (accessed 5 Jan 2004).

4 Centers for Disease Control and Prevention. Preliminary clinical description of severe acute respiratory syndrome. MMWR Morb Mortal Wkly Rep 2003;52(12):255-6.

5 Hsu LY, Lee CC, Green JA, Ang B, Paton NI, Lee L, et al. Severe acute respiratory syndrome (SARS) in Singapore: Clinical features of index patient and initial contacts. CDC. Emerg Infect Dis 2003;9(6):108.

6 Donelly CA, Ghani AC, Leung GM, Hedley AJ, Fraser C, Riley S, et al. Epidemiological determinants of spread of causal agent of severe acute respiratory syndrome in Hong Kong. Lancet 2003;361:1761-6.

7 Poutaren SM, Low DE, Henry B, Finkelstein S, Rose D, Green K, Tellier R, et al. identification of severe acute respiratory syndrome in Canada. N Engl J Med 2003;348:1995-

8 Tsang KW, Ho PL, Ooi GC, Yee WK, Wang T, Chan-Yeubg M, et al. A cluster of cases of severe acute respiratory syndrome in Hong Kong. N Engl J Med 2003;348:1977-85.

9 Lee N, Hui D, Wu A, Chan P, Cameron P, Joynt GM, et al. A major outbreak of severe acute respiratory syndrome in Hong Kong. N Engl J Med 2003;348:1986-94.

10 Twu SJ, Chen TJ, Chen CJ, Olsen SJ, Lee LT, Fisk T, et al. Control of measures of severe acute respiratory syndrome (SARS) in Taiwan. CDC. Emerg Infect Dis 2003;9:1-6. (Accepted 6 November 2003)

doi 10.1136/bmj. .37939 .465729 .44

Health Screening Department, Adult Health Division, Health Promotion Board, 3 Second Hospital Avenue \#06-00, Singapore 168937

Khuan Yew Chow registrar

Casemix Project Office, Planning and Development Division, Ministry of Health Singapore, College of Medicine Building, 16 College Road, Singapore 169854 Chien Earn Lee head 
Quality Management, Singapore General Hospital, Outram Road, Singapore 169608

Moi Lin Ling director

Ear, Nose, and Throat Department, Singapore General Hospital Soon Ghee Yap nursing officer
Clinical Trials and Epidemiology Research Unit, 226 Outram Road Blk A \#03-01, Singapore 169039

Derrick Mok Kwee Heng head of epidemiology

Correspondence to: Moi Lin Ling gptlml@sgh.com.sg 